\title{
ON THE PROJECTIVE VARIETIES ASSOCIATED WITH SOME SUBRINGS OF THE RING OF THETANULLWERTE
}

\author{
RICCARDO SALVATI MANNI
}

\section{Introduction}

Let $(X, L)$ be a principally polarized abelian variety (ppav) of dimension $g$ such that $L$ is a symmetric line bundle, i.e. $i^{*} L \simeq L$ where $i$ is the inversion map $i(x)=-x$. We shall denote by $X[2]$ the two torsion points of $X$ which are fixed by $i$. For any $x$ in $X[2]$ we have an isomorphism

$$
t_{x}^{*}(L)^{2} \simeq L^{2}
$$

Here $t_{x}$ is the translation map.

Let $\tau$ be a point of the Siegel upper half space $\mathbf{H}_{g}$ and $X$ be the abelian variety $\mathbf{C}^{g} /\left(\tau \mathbf{Z}^{g}+\mathbf{Z}^{g}\right)$. As symmetric line bundle $L$ we take $\mathbf{C}^{g} \times \mathbf{C} /\left(\tau \mathbf{Z}^{g}+\mathbf{Z}^{g}\right)$ with the action $(\tau a+b)(z, w)=\left(z+\tau a+b, \mathbf{e}\left(-(1 / 2)^{t} a \tau a+{ }^{t} a z\right) w\right)$.

Here $\mathbf{e}(t)$ stands for $\exp (2 \pi i t)$.

For $x=(1 / 2)\left(\tau m^{\prime}+m^{\prime \prime}\right)$ in $X[2], t_{x}^{*} L$ is still symmetric and we have that the theta function of characteristic $m$ and modulus $\tau$ :

$$
\vartheta_{m}(\tau, z)=\sum_{p \in \mathbf{Z}^{g}} \mathbf{e}\left((1 / 2)^{t}\left(p+\frac{m^{\prime}}{2}\right) \tau\left(p+\frac{m^{\prime}}{2}\right)+{ }^{t}\left(p+\frac{m^{\prime}}{2}\right)\left(z+\frac{m^{\prime \prime}}{2}\right)\right)
$$

is up to a multiplicative constant the unique section of the above line bundle. Here $m^{\prime}$ and $m^{\prime \prime}$ are in $\{0,1\}^{g}$.

Sometime, if it will be necessary, we shall write $\vartheta_{m}(\tau, z)=\vartheta\left[\begin{array}{l}m^{\prime} \\ m^{\prime \prime}\end{array}\right](\tau, z)$.

It is a well known fact that a basis of $H^{0}\left(X, L^{2}\right)$ is given by the $2^{g}$ theta function $\vartheta\left[\begin{array}{c}m^{\prime} \\ 0\end{array}\right](2 \tau, 2 z)$ and from (1) we have theta relation

(3) $\vartheta_{m}(\tau, z)^{2}=\sum_{\sigma} \mathbf{e}\left((1 / 2)^{t}\left(m^{\prime}+\sigma\right) m^{\prime \prime}\right) \vartheta\left[\begin{array}{l}\sigma \\ 0\end{array}\right](2 \tau, 0) \vartheta\left[\begin{array}{c}m^{\prime}+\sigma \\ 0\end{array}\right](2 \tau, 2 z)$.

Received August 24, 1992. 
Let $q$ be an even positive integer we shall denote by $\Gamma_{g}(q)$ the subgroup of $\Gamma_{g}=S p(g, \mathbf{Z})$ defined by

$$
\sigma=\left[\begin{array}{ll}
a & b \\
c & d
\end{array}\right] \equiv 1_{2 g} \bmod q
$$

We shall further denote by $\Gamma_{g}(q, 2 q)$ the subgroup of $\Gamma_{g}(q)$ defined by $\operatorname{diag}\left(a^{t} b\right) \equiv \operatorname{diag}\left(c^{t} d\right) \equiv 0 \bmod 2 q$.

Evaluating at $z=0$ the theta function $\vartheta_{m}(\tau, z)$ we get an holomorphic function $\vartheta_{m}=\vartheta_{m}(\tau)$ defined on $\mathbf{H}_{g}$, that is not identically zero if and only if the characteristic $m$ is even, i.e. ${ }^{t} m^{\prime} m^{\prime \prime} \equiv 0 \bmod 2$. We have exactly $2^{g-1}\left(2^{g}+1\right)=N+$ 1 even characteristics. We know, cf [6] and [8], that the maps $\Theta, \Theta^{2}, \Theta^{4}$ sending $\tau$ in $\left(\cdots \vartheta_{m}(\tau) \cdots\right),\left(\cdots \vartheta_{m}^{2}(\tau) \cdots\right),\left(\cdots \vartheta_{m}^{4}(\tau) \cdots\right)$ from $\mathbf{H}_{g}$ to $\mathbf{P}^{N}$ factorize over $\Gamma_{g}(4,8), \Gamma_{g}(2,4)$ and $\Gamma_{g}(2)$ respectively.

We know that all these maps are injective, cf [6] and [8].

Here we shall show that the injectivity of these maps extends to the Satake compactifications and we shall characterize the points of these compactifications in terms of vanishing of Thetanullwerte. Moreover we shall show that the characterization of the reducible points, obtained in this way, is ideal-theoretic. Under $\Theta$, $\Gamma_{g}(4,8) \backslash \mathbf{H}_{g}$ is biholomorphic onto its image, cf [6]; $\Theta^{2}$ and $\Theta^{4}$ have the same property when $g$ is less or equal to 2, cf [5]. We shall show that $\Theta^{2}$ and $\Theta^{4}$ are not immersions for $g \geq 4$ and that $\Theta^{2}$ is an immersion when $g$ is 3 . This result still holds when we extend $\Theta^{2}$ to the Satake compactification of $\Gamma_{3}(2,4) \backslash \mathbf{H}_{3}$.

We are grateful to G. Lupacciolu and A. Silva for helpful discussions.

\section{Satake compactification}

Let $R$ be an associative ring with the unity, then we shall denote by $M_{p, q}(R)$ the $R$-module of the $p$ by $q$ matrices with coefficients in $R$. Let $A$ and $B$ be in $M_{p, q}(R)$ and $M_{s, t}(T)$ respectively. Then we define the tensor product of matrices

$$
A \otimes B=\left[\begin{array}{ccc}
A b_{11} & \cdots & A b_{1 t} \\
\cdots & \cdots & \cdots \\
A b_{s 1} & \cdots & A b_{s t}
\end{array}\right]
$$

Among all the properties of the tensor product we recall that if rank $A=p$ and rank $B=s$, then rank $A \otimes B=p s$. We shall write $M_{p}(R)$ for $M_{p, p}(R) . S^{g}(R)$ shall denote the submodule of the symmetric matrices of $M_{p}(R) . G L(p, R)$ shall denote the subset of $A$ in $M_{p}(R)$ such that $\operatorname{det} A$ is invertible. We shall de- 
note by $1_{p}$ the unit matrix; a matrix $\sigma$ in $G L(2 g, R)$ is in $S p(g, R)$ if

$$
{ }^{t} \sigma\left[\begin{array}{cc}
0 & 1_{g} \\
-1_{g} & 0
\end{array}\right] \sigma=\left[\begin{array}{cc}
0 & 1_{g} \\
-1_{g} & 0
\end{array}\right]
$$

We shall express $\sigma$ in $S p(g, R)$ as

$$
\sigma=\left[\begin{array}{ll}
a & b \\
c & d
\end{array}\right]
$$

where $a, b, c, d$ are in $M_{g}(R)$.

We know that $S p(g, \mathbf{R})$ acts transitively on $\mathbf{H}_{g}$ as

$$
\sigma \cdot \tau=(a \tau+b)(c \tau+d)^{-1} .
$$

Let $\Gamma$ be a subgroup of finite index of $\Gamma_{g}$, an analytic function $\psi$ defined on $\mathbf{H}_{\boldsymbol{g}}$, which satisfies the functional equation

$$
\phi(\sigma \cdot \tau)=\operatorname{det}(c \tau+d)^{k} \phi(\tau)
$$

for every $\sigma$ in $\Gamma$ (plus a condition at the cusps for $g=1$ ) is called a modular form of weight $k$ (positive, half-integer) relative to $\Gamma$. The set of such functions form a finite dimensional subspace over $\mathbf{C}$ that we shall denote by $[\Gamma, k]$.

The graded ring

$$
A(\Gamma)=\underset{0 \leq k<\infty}{\bigoplus}[\Gamma, k]
$$

is a finitely generated over $\mathbf{C}$, integrally closed domain, it is called the ring of modular forms relative to $\Gamma$ and the projective variety Proj $A(\Gamma)$ associated to it is a compactification of $\Gamma \backslash \mathbf{H}_{g}$, in the sense that this is complex-analytically isomorphic to an open set in the Zariski topology of Proj $A(\Gamma)$.

Usually $\operatorname{Proj} A(\Gamma)$ is called the Satake compactification of $\mathbf{H}_{g} / \Gamma$. Let us describe it more in detail, we refer to [2] for an accurate description. Let $\mathbf{S}_{g}$ be the subset of $M_{2 g, g}(\mathbf{C})$ formed by all matrices

$$
W=\left(\begin{array}{l}
W_{1} \\
W_{2}
\end{array}\right), W_{i} \text { in } M_{g}(\mathbf{C})
$$

having the properties: rank $W=g$ and ${ }^{\mathrm{t}} W_{1} W_{2}$ is in $S^{\mathrm{g}}(\mathbf{C})$; the group $G L(g, \mathbf{C})$ acts on $\mathbf{S}_{g}$ by multiplication on the right. Let $\mathbf{G}_{g}=\mathbf{S}_{g} / G L(g, \mathbf{C})$, then it is a closed submanifold of the grassmannian manifold of all $g$ dimensional subspaces of $\mathbf{C}^{2 g}$. 
The map that send $\tau$ in the class $\left[\begin{array}{c}\tau \\ 1_{g}\end{array}\right]$ is an embedding of $\mathbf{H}_{\boldsymbol{g}}$ in $\mathbf{G}_{\boldsymbol{g}}$. $S p(g, \mathbf{C})$ acts on $\mathbf{G}_{g}$ by multiplication on the left; this is the extension of the action (6), in fact we have

$$
\left[\begin{array}{ll}
a & b \\
c & d
\end{array}\right]\left[\begin{array}{c}
\tau \\
1_{g}
\end{array}\right]=\left[\begin{array}{l}
a \tau+b \\
c \tau+d
\end{array}\right]=\left[\begin{array}{c}
\sigma \cdot \tau \\
1_{g}
\end{array}\right]
$$

Let $k \leq g$, then we have an embedding of $\mathbf{H}_{k}$ in $\mathbf{G}_{g}$ via the map

$$
j_{g, k}\left(\tau_{k}\right)=\left[\begin{array}{l}
1_{g} \\
\tilde{\tau}_{k}
\end{array}\right], \tilde{\tau}_{k}=\left[\begin{array}{cc}
\tau_{k}^{-1} & 0 \\
0 & 0
\end{array}\right] .
$$

We remark that in the case $k=g$, this agrees with the previous embedding. We call the image $j_{g, k}\left(\mathbf{H}_{k}\right)$ the $k$-dimensional standard boundary component of $\mathbf{H}_{g}$.

A subset $A$ of $\mathbf{G}_{g}$ is a $k$-dimensional rational boundary component if there exists $\sigma$ in $\Gamma_{g}$ such that $\sigma(A)$ is the $k$-dimensional standard component. We shall write $A_{k}$ for the union of all $k$ dimensional rational boundary components. We set

$$
\bar{A}_{g}=\bigcup_{k=0}^{g} A_{k}
$$

Then it is a well known fact that $\Gamma \backslash \bar{A}_{g} \simeq \operatorname{Proj} A(\Gamma)$. We call $\Gamma \backslash A_{k}$ the $k$-th stratum of Proj $A(\Gamma)$ and shall denote by $\alpha$ the projection map from $\bar{A}_{g}$ to $\operatorname{Proj} A(\Gamma)$. In particular the Satake compactifications $\operatorname{Proj} A\left(\Gamma_{g}(4,8)\right)$, $\operatorname{Proj} A\left(\Gamma_{g}(2,4)\right)$ and $\operatorname{Proj} A\left(\Gamma_{g}(2)\right)$ have that the $k$-th stratum is a disjoint union of a finite number of quasi projective varieties each complex analytically isomorphic to $\Gamma_{k}(4,8) \backslash \mathbf{H}_{k}, \Gamma_{k}(2,4) \backslash \mathbf{H}_{k}, \Gamma_{k}(2) \backslash \mathbf{H}_{k}$ respectively, $k=0,1, \ldots,(g-1)$.

Clearly each pair of isomorphic components are conjugate under the action of $\Gamma_{g}$. Therefore any point in the boundary can be obtained as

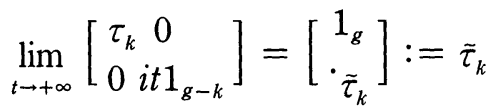

with $\tau_{k}$ in $\mathbf{H}_{k}$ modulo its appropriate relative subgroup $\left(\Gamma_{k}(4,8), \Gamma_{k}(2,4), \Gamma_{k}(2)\right)$. We shall denote by $\alpha_{4,8}, \alpha_{2,4}$ and $\alpha_{2}$ the projection maps from $\bar{A}_{g}$ to Proj $A\left(\Gamma_{g}(4,8)\right)$, Proj $A\left(\Gamma_{g}(2,4)\right)$, Proj $A\left(\Gamma_{g}(2)\right)$ respectively.

Let $m^{\prime}=\left({ }^{t} m_{0}^{\prime},{ }^{t} m_{1}^{\prime}\right)$ and $m^{\prime \prime}=\left({ }^{t} m_{0}^{\prime \prime},{ }^{t} m_{1}^{\prime \prime}\right)$ with $m_{0}^{\prime}, m_{0}^{\prime \prime}$ in $\{0,1\}^{k}$ and $m_{1}^{\prime}, m_{1}^{\prime \prime}$ in $\{0,1\}^{g-k}$, then we shall write $m=m_{0} \oplus m_{1}$ for any $\tau_{k}$ in $\mathbf{H}_{k}$ we put

$$
\vartheta_{m}\left(\tilde{\tau}_{k}\right)=\lim _{t \rightarrow+\infty} \vartheta_{m}\left(\begin{array}{cc}
\tau_{k} & 0 \\
0 & i t 1_{g-k}
\end{array}\right)= \begin{cases}\vartheta_{m_{0}}\left(\tau_{k}\right) & \text { if } m_{1}^{\prime}=0 \\
0 & \text { otherwise }\end{cases}
$$


For any $m$ in $\{0,1\}^{2 g}$ and $\sigma$ in $\Gamma_{g}$, we put

$$
\sigma \cdot m=\left(\begin{array}{cc}
d & -c \\
-b & a
\end{array}\right)\left(\begin{array}{l}
m^{\prime} \\
m^{\prime \prime}
\end{array}\right)+\left(\begin{array}{c}
\operatorname{diag}\left(c^{t} d\right) \\
\operatorname{diag}\left(a^{t} b\right)
\end{array}\right)
$$

Let us denote by $\sigma \circ m$ the unique element in $\{0,1\}^{2 g}$ that is congruent to $\sigma \cdot m$ $\bmod 2$; then (15) give rise to an action of $\Gamma_{g}$ on $\{0,1\}^{2 g}$.

We recall the transformation formula of theta functions; for every $\sigma$ in $\Gamma_{g}$ and characteristic $m$ we have

$$
\begin{aligned}
& \vartheta_{\sigma \circ m}\left(\sigma \cdot \tau,{ }^{t}(c z+d)^{-1} z\right) \\
& =k(\sigma) \operatorname{det}(c \tau+d)^{1 / 2} \mathbf{e}\left(\varphi_{m}(\sigma)\right) \\
& \quad \cdot \mathbf{e}\left((1 / 4)^{t}(\sigma \circ m)^{\prime}(\sigma \cdot m-\sigma \circ m)^{\prime \prime}\right) \mathbf{e}\left((1 / 2)^{t} z(c \tau+d)^{-1} c z\right) \\
& =k(\sigma) \operatorname{det}(c \tau+d)^{1 / 2} \mathbf{e}\left((1 / 2)^{t} z(c \tau+d)^{-1} c z\right) \chi_{m}(\sigma) \vartheta_{m}(\tau, z)
\end{aligned}
$$

where $k(\sigma)$ is an eighth root of unity independent from $m, \tau$ and $z$, and

$$
\varphi_{m}(\sigma)=(-1 / 8)\left({ }^{t} m^{\prime t} b d m^{\prime}-2^{t}{m^{\prime}}^{t} b c m^{\prime \prime}+{ }^{t} m^{\prime \prime} a c m^{\prime \prime}-2^{t} \operatorname{diag}\left(a^{t} b\right)\left(d m^{\prime}-c m^{\prime \prime}\right)\right)
$$

An immediate consequence of (14), (16) and (17) is that the maps

$$
\Theta: \Gamma_{g}(4,8) \backslash \mathbf{H}_{g} \rightarrow \mathbf{P}^{N}, \Theta^{2}: \Gamma_{g}(2,4) \backslash \mathbf{H}_{g} \rightarrow \mathbf{P}^{N}, \Theta^{4}: \Gamma_{g}(2) \backslash \mathbf{H}_{g} \rightarrow \mathbf{P}^{N}
$$

are well defined, extend to the respective Satake compactifications and are $\Gamma_{g} / \Gamma_{g}(4,8), \Gamma_{g} / \Gamma_{g}(2,4), \Gamma_{g} / \Gamma_{g}(2)$ equivariant.

We shall denote by $\bar{\Theta}, \bar{\Theta}^{2}, \bar{\Theta}^{4}$ these extensions.

Clearly the images of these maps are the projective varieties Proj $\mathbf{C}\left[\vartheta_{m}\right]$, Proj $\mathbf{C}\left[\vartheta_{m}^{2}\right]$ Proj $\mathbf{C}\left[\vartheta_{m}^{4}\right]$. We recall that the map $\bar{\Theta}^{2}$ can be obtained, up to a projectivity, composing the map.

$$
\begin{aligned}
\bar{\Theta}_{2}: \operatorname{Proj} A\left(\Gamma_{g}(2,4)\right) & \rightarrow \mathbf{P}^{2^{g}-1} \\
\tau & \rightarrow\left(\cdots \vartheta\left[\begin{array}{c}
m^{\prime} \\
0
\end{array}\right](2 \tau) \cdots\right)
\end{aligned}
$$

with the Veronese map; therefore $\operatorname{Proj} \mathbf{C}\left[\vartheta_{m}^{2}\right] \cong \operatorname{Proj} \mathbf{C}\left[\vartheta\left[\begin{array}{c}m^{\prime} \\ 0\end{array}\right]\right]$.

\section{Fiber of the projection maps}

In this section we shall use several times results obtained in [5] and [8]. Let us recall some of them. We set $C=\Gamma_{g}(2) / \Gamma_{g}(2,4)$ and $G^{\prime}=\Gamma_{g}(2,4) / \Gamma_{g}(4,8)$.

If $i, j$ are distinct positive integer at most equal to $g$, we replace the $(i, j)$ 
coefficient of $1_{g}$ by 2 . We take this matrix as $a$, we put $b=c=0, d={ }^{t} a^{-1}$, the corresponding matrix $A_{i j}$ is an element of $\Gamma_{g}(2,4)$. If $1 \leq i \leq g$ we replace the $(i, i)$ coefficient of $1_{g}$ by -1 . We take this matrix as $a$, we put $b=c=0, a=$ $d$, the corresponding matrix $A_{i i}$ is in $\Gamma_{g}(2,4)$. If $1 \leq i \leq g$ we replace the $(i, i)$ coefficient of the matrix 0 by 2 . We take this matrix as $b$, we put $a=d=1_{g}$, $c=0$; the corresponding matrix $B_{i i}$ is in $\Gamma_{g}(2)$. If $1 \leq i<j \leq g$ we replace the $(i, j)$ and the $(j, i)$ coefficient of the 0 matrix by 2 . We take this matrix as $b$, we put $a=d=1_{g}, c=0$; the corresponding matrix $B_{i j}$ is in $\Gamma_{g}(2,4)$. Finally we set $C_{i j}={ }^{t} B_{i j}$. We recall from [5] the following fact. The $g(2 g+1)$ matrices $A_{i j}$ $(1 \leq i \leq j \leq g), B_{i j}, C_{i j}(1 \leq i<j \leq g), B_{i i}^{2}, C_{i i}^{2}(1 \leq i \leq g)$ are a basis of $G^{\prime}$; the $2 g$ matrices $B_{i i}, C_{i i}(1 \leq i \leq g)$ are a basis of C. Moreover in [8] we proved that the group of characters $\hat{G}^{\prime}$ and $\hat{C}$ are generated by all $\chi_{m}$ and $\chi_{m}^{2}$ respectively, $m$ arbitrary characteristic.

One can deduce the definition of $\chi_{m}$ from (16).

These facts are used to prove the injectivity of the map $\Theta^{2}$ and $\Theta^{4}$, in fact they conduce to the injectivity of $\Theta$ of [8] Theorem 3. Since $\bar{\Theta}$ is injective of [7], using the same method used in the above cited theorem we get

THEOREM 1. The maps $\bar{\Theta}^{2}$ and $\bar{\Theta}^{4}$ are injective.

We have the following diagram

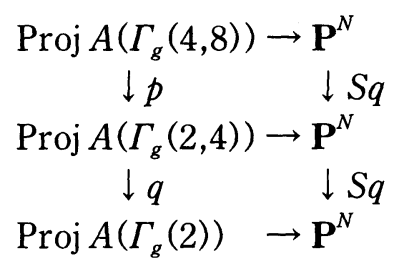

where the horizontal arrows are injective, $S q$ is the map squaring the coordinates and $p$ and $q$ are the usual projection map. We are interested in computing the cardinality of the fiber of the maps $p$ and $q$.

For any point $P$ in $\operatorname{Proj} A\left(\Gamma_{g}(4,8)\right)$ and $Q$ in $\operatorname{Proj} A\left(\Gamma_{g}(2,4)\right)$ we define $S t_{P}$ and $F_{Q}$ as the subgroups of $G^{\prime}$ and $C$ fixing the point $P$ and $Q$ respectively.

We define $\hat{H}_{P}$ and $\hat{D}_{Q}$ as the subgroup of $\hat{G}^{\prime}$ resp. $C^{\prime}$ generated by all $\chi_{m} \chi_{n}$, $\chi_{m}^{2} \chi_{n}^{2}$ respectively such that the product $\vartheta_{m} \vartheta_{n}$ doesn't vanish at $\alpha_{4,8}^{-1}(P)$ and $\alpha_{2,4}^{-1}(Q)$ resp. In [8] we remarked that $S t_{P}$ and $\hat{H}_{P}$ are dual and the same is true for $F_{Q}$ and $\hat{D}_{Q}$ moreover we proved that, for the points of the $g$-th stratum, we have $\# q^{-1}(R)=\# \hat{D}_{Q}$ for any $Q$ such that $q(Q)=R$. This was a consequence of the proof of Theorem 3 . 
Therefore, now as consequence of Theorem 1, we can extend this result to all points of the Satake compactifications. We can prove the following

TheOREM 2. Let $R$ denote a point of the $k$-th stratum of $\operatorname{Proj} A\left(\Gamma_{g}(2)\right)$; then we have

$$
\# q^{-1}(R)=2^{g+k} .
$$

Proof. We have to show that $\# \hat{D}_{Q}=2^{g+k}$ for any $Q$ in the $k$-th stratum of $\operatorname{Proj} A\left(\Gamma_{g}(2,4)\right)$. Already in [8] we proved the statement relatively to the $g$-th stratum. In view of Lemma 10 of [8] that states $\hat{D}_{\sigma \cdot Q}=\hat{D}_{Q}^{\sigma}$ we can assume in $a_{2}^{-1}(R)$ points of the form $(13)$ and $\vartheta_{0}\left(\tilde{\tau}_{k}\right) \neq 0$. Therefore to prove the proposition, it is enough to find $g+k$ linearly independent $(\bmod 2)$ characteristics whose associate Thetanullwerte don't vanish at $\hat{\tau}_{k}$ cf [8]. We know that there are $2 k$ linearly independent characteristics $n_{1}, \ldots, n_{2 k}$ in $\{0,1\}^{2 k}$ such that $\vartheta_{n}\left(\tau_{k}\right) \neq 0$ $1 \leq i \leq 2 k$. Let $m=n_{i} \oplus m_{1}, 1 \leq i \leq 2 k$ and $m_{1}^{\prime}=0$, then we have $\vartheta_{m}\left(\tilde{\tau}_{k}\right) \neq 0$ for all characteristics $m$ of the above form, thus we can get at least $(g+k)$ linearly independent characteristics such that the associate Thetanullwerte don't vanish at $\hat{\tau}_{k}$. Using (10) it is immediate that all $B_{\imath i}, k+1 \leq i \leq g$, belong to $F_{Q}$, therefore $\# F_{Q} \geq 2^{g-k}$, thus we get $\# \hat{D}_{Q}=2^{g+k}$.

For studying the fiber of the map $p$ we need a refinement of the decomposition (12). Let $\underline{r}=\left(r_{1}, r_{2}, \ldots, r_{h}\right)$ be a partition of $\left.k \leq g\right)$, we put

$$
\mathbf{H}_{\underline{r}}=j_{g, k}\left(\mathbf{H}_{i_{1}} \times \mathbf{H}_{r_{2}} \times \cdots \times \mathbf{H}_{r_{h}}\right)
$$

and

$$
A_{\underline{r}}=\Gamma_{g} \cdot \mathbf{H}_{\underline{r}}
$$

let $\underline{s}$ be a refinement of $\underline{r}$, we shall write $\underline{s}<\underline{r}$, then we have $A_{\underline{s}} \subseteq A_{\underline{r}}$ if and only if $\underline{s}<\underline{r}$.

Clearly $A_{k}$ is equal to $A_{(k)}$. For any partition $\underline{r}$ we shall denote by $A_{\underline{r}}^{0}$ the set of points in $A_{\underline{r}}$ that are not contained in any refinement of $\underline{r}$, then we have

$$
A_{k}=\dot{U}_{\underline{r}, \text { partition of } k} A_{\underline{\underline{r}}}^{0}
$$

We call $A_{(g)}^{0}$ the set of irreducible points of $A_{g}=\mathbf{H}_{g}, A_{g} \backslash A_{g}^{0}$, the set of reducible points and $\Gamma \backslash A_{\underline{r}}^{0}$ the $\underline{r}$ component of the $k$-th stratum.

Let $\underline{s}=\left(s_{1}, \ldots, s_{l}\right), 1 \geq h$ be a partition of $j<k$, then we shall write $\underline{s}<<\underline{r}$ if there exist $k$ indices $0=i_{0}<i_{1}<i_{2}<\ldots i_{k}=l$ such that $s_{i_{\alpha}}+s_{i_{\alpha}+1}$ 
$+\cdots+s_{i_{\alpha+1}}<r_{a+1}, 0 \leq \alpha \leq k-1$.

We put $\overline{\mathbf{H}}_{\underline{\underline{r}}}=\mathbf{H}_{\underline{\underline{r}}} \cup \underset{\underline{s}<<\underline{\underline{r}}}{\cup} \mathbf{H}_{\underline{\underline{s}}}$.

THEOREM 3. Let $Q$ denote a point of the $\underline{r}=\left(r_{1}, r_{2}, \ldots r_{h}\right)$ component of the $k$-th stratum of Proj $A\left(\Gamma_{g}(2,4)\right)$; then we have

$$
\# p^{-1}(Q)=2^{(1 / 2)(g+k)(g+k+1)-h} .
$$

In [8] we proved the theorem relatively to points in the $g$-th stratum. The same method, using the results of Theorem 2, extends to all other strata reducing ourselves to computing the cardinality of the fiber over a point of the $g$-th stratum. In this case we obtain $\# p^{-1}(Q)=2^{g(2 g+1)-h}$. At this point the proof is a consequence of that of Proposition 4 of [8] and of the decomposition $(1 / 2)(g+k)$ $(g+k+1)-h=(k(2 k+1)-h)+\left(\begin{array}{c}g-k+1 \\ 2\end{array}\right)+2 k(g-k)$.

Let $x_{1}, x_{2}, \ldots, x_{h}, x_{h+1}$ be characteristics in $\{0,1\}^{2 r_{1}}, \ldots\{0,1\}^{2 r_{h}},\{0,1\}^{2(g-k)}$ respectively then we put

$$
m=x_{1} \oplus x_{2} \ldots \oplus x_{h+1}
$$

If one of the $x_{i}, 1 \leq i \leq h$, is an odd characteristic or $x_{h+1}^{\prime} \neq 0$, then it is a well known fact that the associate Thetanullwerte vanish on $\mathbf{H}_{\underline{r}}$. Let $\bar{V}_{x}$ be the subset of $\bar{A}_{g}$ defined by the vanishing of all above described Thetanullwerte, then generalizing Theorem 5 of [8] we have

THEOREM 4. $\quad \bar{V}_{\underline{r}}=\Gamma_{g}(2) \cdot \overline{\mathbf{H}}_{\underline{r}}$

Clearly a similar statement holds for the conjugate components.

From now to the end of this section we shall consider the case $k=g$.

Let as recall from [6] p. 187 the heat equation; differantiating the theta functions $\vartheta_{m}(\tau, z)$ respect to $z_{1}, \ldots, z_{g}, \tau_{11}, \tau_{12}, \ldots, \tau_{g g}$ we have

$$
\begin{gathered}
(2 \pi i)^{p} \prod_{k=1}^{p}\left(\delta_{i_{k}}^{j_{k}}+1\right) \partial^{p} \vartheta_{m} / \partial \tau_{i_{1} j_{1}} \cdots \partial \tau_{i_{p} j_{p}} \\
=\partial^{2 p} \vartheta_{m}(\tau, z) / \partial z_{i_{1}} \partial z_{j_{1}} \cdots \partial z_{i_{p}} \partial z_{j_{p}} .
\end{gathered}
$$

Moreover we know that at all point of $\mathbf{H}_{g}$

$$
\operatorname{rank}^{t}\left(\partial \vartheta_{m}(\tau, 0) / \partial z_{1}, \ldots, \partial \vartheta_{m}(\tau, 0) / \partial z_{g}\right)_{m \text { odd }}=g
$$

THEOREM 5. i) $V_{\underline{r}}=\bar{V}_{\underline{r}} \cap \mathbf{H}_{g}$ is a complex submanifold of $\Gamma_{g}(4,8) \backslash \mathbf{H}_{g}$. 
ii) $\Gamma_{g}(4,8) \backslash V_{\underline{r}}$ is a complex submanifold of $\Gamma_{g}(4,8) \backslash \mathbf{H}_{g}$.

Proof. It is clear that (ii) descends from (i). Let us prove (i). We know that set theoretically $V_{\underline{r}}=\Gamma_{g}(2) \mathbf{H}_{\underline{r}}$, therefore we have codim $V_{\underline{r}}=\Pi_{1 \leq i<j \leq h} r_{i} r_{j}$.

We shall show that this number is the rank of the matrix

$$
\left(\partial \vartheta_{m}(\tau, 0) / \partial \tau_{i j}\right)_{1 \leq i \leq j \leq g},
$$

with $m$ of the form (25) and $\tau$ in $V_{\underline{r}}$. Applying the transformation formula (16) we can assume $\tau$ in $\mathbf{H}_{\underline{r}}$, i.e.

$$
\tau=\left[\begin{array}{cccc}
\tau_{1} & 0 & . & 0 \\
0 & \tau_{2} & 0 & . \\
. & \cdot & . & 0 \\
0 & . & 0 & \tau_{h}
\end{array}\right], \tau_{i} \in \mathbf{H}_{r_{i}}
$$

We know that all $\partial \vartheta_{m}(\tau, 0) / \partial \tau_{i j}$ are 0 if more than two $x_{i}$ 's are odd. Let us assume $x_{1}$ and $x_{2}$ odd characteristics in the decomposition (25), then we have

$$
\partial \vartheta_{m}(\tau, 0) / \partial \tau_{i j}=\left\{\begin{array}{l}
\prod_{i=3}^{h} \vartheta_{x_{i}}\left(\tau_{i}\right)\left(\partial \vartheta_{x_{1}}\left(\tau_{1}, 0\right) / \partial z_{i}\right)\left(\partial \vartheta_{x_{2}}\left(\tau_{2}, 0\right) / \partial z_{j}\right) \\
\quad \text { if } 1 \leq i \leq r_{1}<j \leq r_{1}+r_{2} \\
0 \quad \text { otherwise. }
\end{array}\right.
$$

Let us take $x_{3}, \ldots, x_{h}$, even characteristics in such way that

$$
\prod_{i=3}^{h} \vartheta_{x_{i}}\left(\tau_{i}\right) \neq 0
$$

This is always possible; now if we allow to the $x_{i}$ 's of varying in the set of odd resp. even characteristics according as $i \leq 2$ or $i \geqslant 3$; we get a submatrix $A_{12}$ of (28). It is an immediate consequence of (27) and of the tensor product of matrices that this submatrix has rank $r_{1} r_{2}$. Iterating this process we obtain similar matrices $A_{i j}, 1 \leq i \leq j \leq g$, of rank $r_{i} r_{j}$. We remark that the matrix (28) is of the form

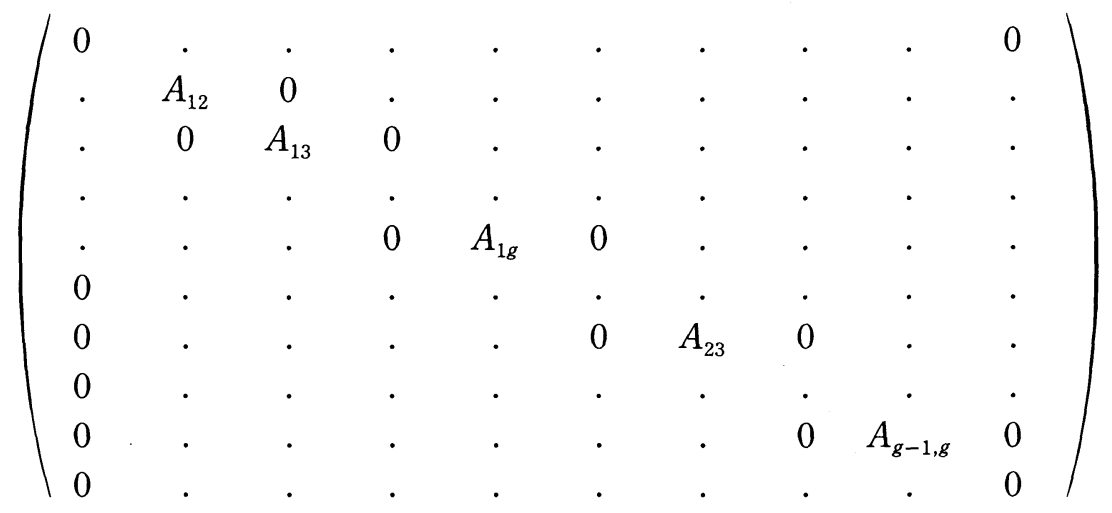


that has the required rank. In this case too a similar statement holds for conjugate components. Finally we remark that all irreducible component of $V_{\underline{r}}$ are two by two disjoint.

\section{Comparison of projective varieties}

We shall prove that in genus 3 case $\operatorname{Proj} \mathbf{C}\left[\vartheta_{m}^{2}\right]$ is a normal projective variety, therefore $\bar{\Theta}^{2}$ will be an immersion.

It is a well known fact, cf. [2] and [3] that, for $g \geq 3$, the set of regular points of $\operatorname{Proj} A\left(\Gamma_{g}(2,4)\right)$ coincides with. $\Gamma_{g}(2,4) \backslash A_{g}^{0}$, moreover at these points we have that the rank of

$$
\left(\vartheta\left[\begin{array}{c}
m^{\prime} \\
0
\end{array}\right](2 \tau, 0), \partial \vartheta\left[\begin{array}{c}
m^{\prime} \\
0
\end{array}\right](2 \tau, 0) / \partial \tau_{11}, \ldots, \partial \vartheta\left[\begin{array}{c}
m^{\prime} \\
0
\end{array}\right](2 \tau, 0) / \partial \tau_{g g}\right)_{m^{\prime} \in\{0,1\}^{g}}
$$

is $(1 / 2) g(g+1)+1=\operatorname{dim} \operatorname{Proj} A\left(\Gamma_{g}(2,4)\right)+1$; then $\Theta^{2}$ is an immersion at the regular points.

Theorem 5. Let us assume $g=3$, then Proj $\mathbf{C}\left[\vartheta_{m}^{2}\right]$ is a normal variety, therefore $\bar{\Theta}^{2}$ is an immersion.

Proof. We have Proj $\mathbf{C}\left[\vartheta_{m}^{2}\right] \simeq \operatorname{Proj} \mathbf{C}\left[\vartheta\left[\begin{array}{l}m^{\prime} \\ 0\end{array}\right]\right]$. It is an hypersurface of degree 16 in $\mathbf{P}^{7}$ cf [3], therefore it is Cohen-Macaulay. Since the subvariety of the singular points has codimension 2 , it is regular in codimension 1 , thus it is normal cf. [4] p. 187. As B. Runge pointed out [9] since $\operatorname{Proj} \mathbf{C}\left[\vartheta\left[\begin{array}{l}m^{\prime} \\ 0\end{array}\right]\right]$ is a complete intersection, this variety is projectively normal.

Now, we want to disprove that $\Theta^{2}$ and $\Theta^{4}$ are immersions when $g \geq 4$; then we shall consider these maps at some special point; in particular let $\tau_{0}$ be equal to the diagonal matrix

$$
\tau_{0}=\left[\begin{array}{cccc}
\tau_{11} & 0 & . & 0 \\
0 & \tau_{22} & . & . \\
. & . & . & 0 \\
0 & . & 0 & \tau_{g g}
\end{array}\right]
$$

We put $Q_{0}=\alpha_{2,4}\left(\tau_{0}\right)$ and $R_{0}=\alpha_{0}\left(\tau_{0}\right)$. Let $D$ be the finite group generated by $A_{i i}, 1 \leq i \leq g$. It is a consequence of [8] that all $\sigma$ in $\Gamma_{g}(2)$ fixing $\tau_{0}$ are the elements of $D$. Moreover there exists $\sigma_{1}$ in $S p(g, \mathbf{R})$ such that $\sigma_{1}$ centralizes all ele- 
ments of $D$ and $\sigma_{1} \cdot \tau_{0}=i 1_{g}$. Let

$$
\sigma_{2}=\left(\begin{array}{cc}
1_{g} & -i 1_{g} \\
0 & 1_{g}
\end{array}\right)
$$

then $\sigma=\sigma_{2} \cdot \sigma_{1}$ sends $\tau_{0}$ to 0 in $S^{g}(\mathbf{C})$ and fix all elements of $D$. Clearly $\sigma$ is an analytic automorphism of $\mathbf{G}_{\boldsymbol{g}}$.

Let $x$ be an element of $D$, then it is of the form $a=d=y, b=c=0$, with $y$ diagonal matrix with -1 and 1 as entries.

Let $A$ be in $S^{g}(\mathbf{C})$, then the action of $x$ at a neighborhood of 0 is given by

$$
x \cdot A=y A y .
$$

Let $Y$ be the quotient space $S^{g}(\mathbf{C}) / D$ and let us consider all monomials of the form

$$
a_{i_{1} i_{2}} a_{i_{2} i_{3}} \cdots a_{i_{n} i_{1}} \quad 1 \leq i_{1}, i_{2}, i_{3}, \ldots, i_{n} \leq g,
$$

they are invariant for the actions of $D$ on $S^{g}(\mathbf{C})$. Let us denote by $m$ the maximal ideal of the homomorphic local ring $O_{0, Y}$.

Lemma 1. i) The dimension of the tangent space $T_{0} Y$ at 0 of $Y$ is $t_{g}=$ $g\left(\begin{array}{l}g \\ 2\end{array}\right)+(1 / 2) \sum_{h=3}^{g}(h-1) !\left(\begin{array}{l}g \\ h\end{array}\right)$.

ii) A basis of $m / m^{2}$ consists of all monomials of the form (34) of degree $\leq g$ with the indices appearing with multiplicity 2.

Proof. (i) follows from (ii), since there is a correspondence between $k$ cycles of $g$ elements and the monomials decribed in (ii). Moreover two cycles determine the same monomial if and only if they are one the inverse of the other. Thus we get $\operatorname{dim}_{\mathrm{C}} T_{0} Y=t_{g}$.

(ii) is a consequence of the fact that these monomials are a minimal set of generators for the ring of the invariants $\mathbf{C}\left[\mathrm{a}_{i j}\right]^{D} 1 \leq i \leq j \leq g$.

Clearly $\sigma$ induces an isomorphism between

$$
T_{R_{0}} \operatorname{Proj} A\left(\Gamma_{g}(2)\right), T_{Q_{0}} \operatorname{Proj} A\left(\Gamma_{g}(2,4)\right) \text { and } T_{0} Y \text {. }
$$

We have

THEOREM 6 . i) $\Theta_{2}$ is not a biholomorphic map when $g \geq 4$

ii) $\Theta^{4}$ is not a biholomorphic map when $g \geq 4$. 
Proof. i) We know that a holomorphic map $\varphi: W \rightarrow Z$ is an immersion at $P$ in $W$ if and only if the jacobian map

$$
T_{P} \varphi: T_{P} W \rightarrow T_{\varphi(P)} Z
$$

is injective cf. [1]. Let us prove (i). Clearly in this case we have $\operatorname{dim} T_{\varphi\left(Q_{0}\right)} Z \leq$ $2^{g}-1$ and

$$
t_{g}=t_{g-1}+g+(1 / 2) \sum_{h=2}^{(g-1)} h !\left(\begin{array}{c}
g-1 \\
h
\end{array}\right)>t_{g-1}+2^{g-1}
$$

for $g \geq 4$. Moreover $t_{3}=7$; this proves (i).

The method, that we shall use to prove (ii), works for the first case too, but, as we shall see, it is less elegant.

We have to write explicitly part of the matrix associated to the jacobian map. We have to consider the $t_{g}$ by $(N+1)$ matrix

$$
\left(\partial^{n} \vartheta_{m}^{4}(\tau) / \partial \tau_{i_{1} i_{2}} \cdots \partial \tau_{i_{n} i_{1}}\right)_{m \text { even }}
$$

where $\partial \tau_{i_{1} i_{2}} \cdots \vartheta \tau_{i_{n} i_{1}}$ is the basis of $T_{R_{0}} \operatorname{Proj} A\left(\Gamma_{g}(2)\right)$ related to the basis of $T_{0} Y$. We shall say that a characteristic $m$ is of type 0 if ${ }^{t} m^{\prime} m^{\prime \prime}=0$. Using the heat equations (26) and evaluating at $z=0$ and $\tau=\tau_{0}$ an elementary and tedious computation gives us

$$
\begin{aligned}
4 \pi^{4} \partial^{4} \vartheta_{m}^{4}\left(\tau_{0}, 0\right) / \partial \tau_{12} \partial \tau_{23} \partial \tau_{34} \partial \tau_{41} \\
\quad=\left\{\begin{array}{l}
\vartheta_{m}^{3}\left(\tau_{0}\right) \partial^{8} \vartheta_{m}\left(\tau_{0}, 0\right) /\left(\partial z_{1}\right)^{2}\left(\partial z_{2}\right)^{2}\left(\partial z_{3}\right)^{2}\left(\partial z_{4}\right)^{2} \\
\text { if } m \text { is of type } 0 \\
0 \quad \text { otherwise }
\end{array}\right.
\end{aligned}
$$

We remark that

$$
\partial^{4} \vartheta_{m}\left(\tau_{0}, 0\right) / \partial \tau_{12} \partial \tau_{23} \partial \tau_{34} \partial \tau_{41}=\partial^{4} \vartheta_{m}\left(\tau_{0}, 0\right) / \partial \tau_{12} \partial \tau_{24} \partial \tau_{43} \partial \tau_{13}
$$

for all characteristics $m$; therefore the matrix (38) has rank less than $t_{g}$. This proves the theorem.

\section{REFERENCES}

[1] Fischer G.; Complex Analytic Geometry, Lecture Notes in Math., 538, SpringerVerlag (1976).

[2] Freitag E.; Siegelsche Modulfunktionen, Die Grund. der Math. Wiss., 254, Springer-Verlag (1983).

[3] van Geemen B., van der Geer G.; Kummer Varieties and the Moduli Spaces of Abelian Varieties, Amer. J. Math., 108 (1986), 615-641. 
[4] Hartshorne R.; Algebraic Geometry Graduate Texts in Math, 52, Springer-Verlag (1977).

[5] Igusa J. I.; On Siegel Modular Form of Genus two II. Amer. J. Math., 86 (1964), $392-412$.

[6] —; Theta Functions, Die Grund, der Math. Wiss. 194, Springer-Verlag (1972).

[7] - On the Variety associated with the Ring of Thetanullwerte, Amer. J. Math., 103 (1981), 377-398.

[8] Salvati Manni R.; Moduli Space of ppav with Level (Theta) Structure and Reducible Points, to appear on Amer. J. Math.

[9] Runge B., "On Siegel modular forms", J. reine angew. Math., 443 (1993), 57-85.

Dipartimento dio Matematica

Istituto "Guido Castelnuovo"

Universita degli Studi di Roma

"La Sapienza"

Piassale Aldo Moro, 2

I-00185 Roma, Italy 